Rev. Bras. Saúde Prod. Anim., Salvador, v.17, n.3, p.545-552 jul./set., $2016 \quad$ http://www.rbspa.ufba.br ISSN 15199940

\title{
Metabolismo respiratório e da glicose de Carassius auratus submetidos à concentrações de eugenol
}

\section{Respiratory metabolism of "Carassius auratus" in different concentrations of eugenol}

\author{
HONORATO, Claucia Aparecida ${ }^{1 *}$; NASCIMENTO, Camila Aparecida ${ }^{1}$
}

${ }^{1}$ Centro Universitário da Grande Dourados, Faculdade de Ciências Extas e Agrárias, Curso de Medicina Veterinária, Dourados, Mato Grosso, Brasil.

*Endereço para correspondência: clauciahonorato@yahoo.com.br

\section{RESUMO}

Este trabalho tem como finalidade avaliar o uso do eugenol como anestésico para Carassius auratus mensurando o tempo de indução à anestesia em relação a diferentes concentrações e seus efeitos nas trocas gasosas respiratórias. Os peixes foram expostos as concentrações de 20, 40, 80, 120, $150 \mathrm{mg} \mathrm{L}^{-1}$ de eugenol. Os resultados foram analisados segundo um delineamento inteiramente casualisado (DIC) com seis tratamentos e cinco repetições cada um, submetidos à análise de variância e quando significativos, as médias foram comparadas pelo teste t de Student $(\alpha=0,05)$. Os valores de $\mathrm{PaO}_{2} \mathrm{e} \quad \mathrm{PaCO}_{2}$ foram submetidos à regressão polinomial $(p>0,05)$. Foram avaliados o tempo de sedação e recuperação. $O$ sangue foi retirado para analise parâmetros hematológicos, $\mathrm{pH}$, pressão parcial de oxigênio $\left(\mathrm{PaO}_{2}\right)$, pressão parcial de dióxido de carbono $\left(\mathrm{PaCO}_{2}\right)$, bicarbonato $\left(\mathrm{HCO}_{3}^{-}\right)$e glicose. As concentrações acima de $80 \mathrm{mg} . \mathrm{L}^{-1}$ apresentaram sedação com 62 seg. A recuperação possui correlação inversa ao tempo de anestesia. O aumento das concentrações de eugenol proporcionou elevação de $27,5 \%$ nos hematócrito e de $37 \%$ no eritrócitos e houve aumento na glicose plasmática. Ademais, apresentaram diminuição na pressão de oxigênio no sangue $\left(\mathrm{PaO}_{2}\right)$, e aumento na pressão de dióxido de carbono $\left(\mathrm{PaCO}_{2}\right)$. A utilização do eugenol possibilitaria e melhoria o manejo nas pisciculturas sendo a dose recomendada de $40 \mathrm{mg}$. $\mathrm{L}^{-1}$ para Carassius auratus pois minimiza os riscos inerentes a processos anestésicos rotineiros, com menor comprometimento cardiovascular e, consequentemente, redução do risco de óbito.

Palavras-chave: anestesia, hemogasometria, manejo de peixes

\section{SUMMARY}

The study aimed to evaluate the use of eugenol as an anesthetic for Carassius auratus measuring time to anesthesia induction in different concentrations and their effects on gas exchange breathing. The fish were exposed to concentrations of $20,40,80,120,150 \mathrm{mg} \mathrm{L}-1$ of eugenol. The results were analyzed according to a completely randomized design with six treatments and five replications were submitted to analysis of variance and when significant, averages were compared by Student's test $(\alpha=0,05)$. the probability $5 \% \cdot \mathrm{PaO}_{2} \mathrm{e}$ $\mathrm{PaCO}_{2}$ values were submitted to polynomial regression $(p>0.05)$. The sedation and recovery time it's the evaluation. Blood was withdrawn for hematological parameters analysis, $\mathrm{pH}$, partial pressure of oxygen $\left(\mathrm{PaO}_{2}\right)$, partial pressure of carbon dioxide $\left(\mathrm{PaCO}_{2}\right)$, bicarbonate $\left(\mathrm{HCO}_{-3}\right)$ and glucose. Concentrations above 80 mg.L-1 presented sedation with $62 \mathrm{sec}$. The recovery has inverse correlation to time of anesthesia. Anesthetic induction of: $C$. auratus to different concentrations of eugenol provided 27.5 elevation on the values of percentage of hematocrit and erythrocyte number 37 and an increase in plasma glucose. C. auratus when subjected to the increased concentration of eugenol showed decrease in blood oxygen pressure $\left(\mathrm{PaO}_{2}\right)$, and increase in pressure of carbon dioxide $\left(\mathrm{PaCO}_{2}\right)$. The use of eugenol makes handling the fish farms, the recommended dose is $40 \mathrm{mg}$. $\mathrm{L}^{-1}$ for Carassiusauratus that minimize the risks inherent in routine anesthetic procedures, with less cardiovascular impairment and, therefore, reducing the risk of death.

Keywords: anesthesia, hemogasometry, fish management 


\section{INTRODUÇÃO}

Dentre as espécies de peixes ornamentais com grande popularidade destaca-se Carassius auratus que ocupa lugar de destaque na comercialização mundial em função da sua aparência, docilidade durante o manejo e adaptação ao confinamento (TAGHIZADEH et al., 2013).

Os peixes ornamentais durante o cultivo, frequentemente precisam ser manejados para classificação e comercialização, o que envolve mudanças de ambiente e alteração na sua homeostasia. A utilização de substâncias anestésicas pode diminuir injurias e morte durante as práticas de manejo (GHOLIPOURKANANI et al., 2013). Dentre os anestésicos com potencial para utilização na piscicultura destaca-se o eugenol. Esta substancia é muito usado na odontologia (MAZZAFERA, 2003). $O$ eugenol é recomendado como um anestésico com poucos riscos de intoxicação (INOUE et al., 2011) eficácia, seguridade e de baixo custo (PADUA et al., 2012).

A anestesia em peixes pode ser afetada por fatores biológicos tais como diferença entre espécies e tamanho e por fatores ambientais (ROTILI et al., 2012). Dentre os estágios de anestesia de peixe, observa-se nos primeiros estágios (I e II) a diminuição dos batimentos operculares e no estágio III aumento dos movimentos operculares (ROSS \& ROSS, 2008). Como as brânquias dos peixes têm múltiplas finalidades, desde promover as trocas gasosas, regulação osmótica iônica e hematológica (GHOLIPOURKANANI et al., 2013), a diminuição nos movimentos operculares podem alterar estas funções. A utilização de anestésicos pode causar reações adversas secundárias tais como acidose e estresse osmótico devido à parada respiratória e insuficiente troca gasosa e iônica entre o sangue e a água (ZAHL et al., 2012).

Este trabalho tem como objetivo avaliar o uso do eugenol como anestésico para Carassius auratus mensurando o tempo de indução à anestesia em relação a diferentes doses e seus efeitos nas trocas gasosas respiratórias e no metabolismo energético.

\section{MATERIAL E MÉTODOS}

Os exemplares de Carassius auratus foram mantidos por um período de aclimatação de 7 dias no laboratório. Estes foram acondicionados em um tanque de 100L de água termostatizada e aerada. Os peixes foram alimentados com ração comercial ad libtum (45\% Proteína bruta, $3800 \mathrm{kcal}$. $\mathrm{Kg}^{-1}$ Energia digestível). A qualidade de água foi monitorada diariamente com $\mathrm{O}$ multiparâmetro: para aferição da temperatura $\left(\mathrm{T}^{\mathrm{o}} \mathrm{C}\right)$, do potencial hidrogeniônico $(\mathrm{pH})$ e do oxigênio dissolvido $\left(\mathrm{O}_{2} \mathrm{D}\right)$.

$\mathrm{Na}$ indução anestésico foram separados 60 exemplares de Carassius auratus com peso de $10,53 \pm 1,04 \mathrm{~g}$ divididos em 6 caixas de $20 \mathrm{~L}$ de água termostatizada e aerada. Os peixes foram expostos a concentrações de 20, 40, 80, 120, $150 \mathrm{mg} \mathrm{L}^{-1}$ de eugenol $(\mathrm{O}$ eugenol por ser oleoso, foi diluído em álcool etílico $\left(92,8^{\circ}\right)$ no momento do uso, no que resultou em solução-estoque à concentração de $\left.100 \mathrm{mg} \mathrm{mL}^{-1}(1: 10)\right)$, e um controle (peixes submetidos ao manejo de troca de aquários sem utilização de anestésico). Submetidos individualmente ao banho anestésico, no qual foi monitorado o tempo para a indução anestésica e o tempo de recuperação. $\mathrm{O}$ tempo necessário para a latência total dos peixes foi considerado 
como o início da exposição até a perda total de equilíbrio na coluna de água e a parada dos batimentos operculares, condição equivalente ao estágio III de anestesia (ROSS \& ROSS, 2008). Após atingir o estágio III, retirou-se o sangue com seringa de $1 \mathrm{~mL}$ heparinizada pela veia caudal e transferidos para um aquário com água sem anestésico, onde foi verificado o tempo de recuperação, considerado como o período necessário para que os peixes recuperassem $o$ equilíbrio e a natação ativa na coluna de água.

Para a coleta de sangue cinco peixes de cada tratamento foram coletados aleatoriamente. $\mathrm{O}$ sangue foi analisado em aparelho de hemogasometria. Os parâmetros analisados foram $\mathrm{pH}$, pressão parcial de oxigênio $\left(\mathrm{PaO}_{2}\right)$, pressão parcial de dióxido de carbono $\left(\mathrm{PaCO}_{2}\right)$ e bicarbonato $\left(\mathrm{HCO}_{3}^{-}\right)$e foi analisado a glicose (TRINDER, 1969). Os parâmetros hematimétricos, foram determinados $\mathrm{O}$ percentual do hematócrito, pelo método do microhematócrito (GOLDENFARB et al., 1971). A contagem de eritrócitos após diluição do sangue previamente homogeneizado em solução formolcitrato (1:200) e contagem realizada em hemocitômetro. Para determinação da concentração de hemoglobina, foi utilizado o método da cianometahemoglobina (COLLIER, 1944). Com estes foram calculados os índices hematimétricos (WINTROBE, 1934), compreendidos pelo Volume Corpuscular Médio (VCM) e Concentração de Hemoglobina Corpuscular Média (CHCM).

Os resultados foram analisados segundo um delineamento inteiramente casualisado (DIC) com seis tratamentos e dez repetições para as analises de indução e recuperação anestésica e cinco repetições para as analises sanguíneas, submetidos à análise de variância e quando significativos, as médias foram comparadas pelo teste $t$ de Student a 5\% de probabilidade. Os valores de $\mathrm{PaO}_{2} \mathrm{e}$ $\mathrm{PaCO}_{2}$ foram submetidos à regressão polinomial $(\mathrm{p}>0,05)$.

\section{RESULTADOS E DISCUSSÃO}

As concentrações testadas foram eficientes para induzir a imobilidade de C. auratus com diferentes tempos de indução anestésica (Figura 1a). Durante a indução anestésica, o comportamento dos peixes seguiu o seguinte padrão: hiperatividade ao primeiro contato com o anestésico, caracterizada pela natação agitada contra as paredes do aquário; natação lenta; diminuição dos batimentos operculares, parada do animal na posição dorso ventral, perda da reação aos estímulos externos; perda do tônus muscular. Em nenhuma das concentrações de eugenol testadas houve mortalidade.

O tempo para que o $C$. auratus apresentasse imobilidade foi inversamente proporcional ao aumento na dose de eugenol administrada. A concentração de $20 \mathrm{mg} . \mathrm{L}^{-1}$ obtiveram tempo de indução anestésica em torno de 202seg, considerado longo para atingir o plano anestésico. O tempo de indução anestésica de $77 \pm 18$ seg foi obtida pela concentração de $40 \mathrm{mg} \cdot \mathrm{L}^{-1}$. Alevinos de Pseudopatystoma corruscans apresentam tempos de indução inferiores a um minuto com concentrações de 50, 75 e $100 \mathrm{mg} . \mathrm{L}^{-1}$ de eugenol (VIDAL et al., 2008). A concentração adequada de eugenol para indução e recuperação anestésica em jundiás (Rhamdia voulezi) é de 50mg.L L $^{-1}$ (DIEMER et al., 2012). O óleo de cravo possui efeito anestésico para alevinos de lambari, sendo $50 \mathrm{mg} . \mathrm{L}^{-}$ ${ }^{1}$ a concentração eficiente e segura para indução à anestesia profunda em até 1,5 
minuto, ressaltando a maior resistência devido a diferença de peso em comparação as demais espécies (PEREIRA-DA-SILVA et al., 2009). Carassius auratus com $2,7 \mathrm{~g}$, não

a)

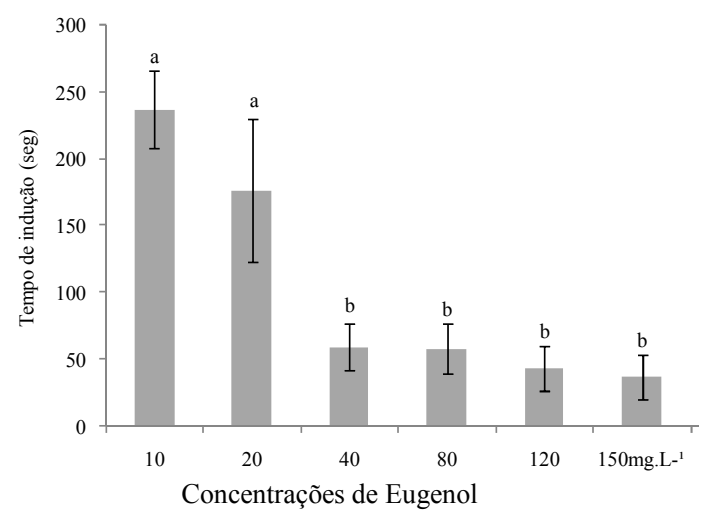

apresenta sedação com doses de eugenol inferiores a $37,5 \mathrm{mg} . \mathrm{L}^{-1}$ e a dose de $75 \mathrm{mg} \mathrm{L}^{-1}$ acarretou em morte de $25 \%$ dos peixes (BITTENCOURT et al., 2012).

b)

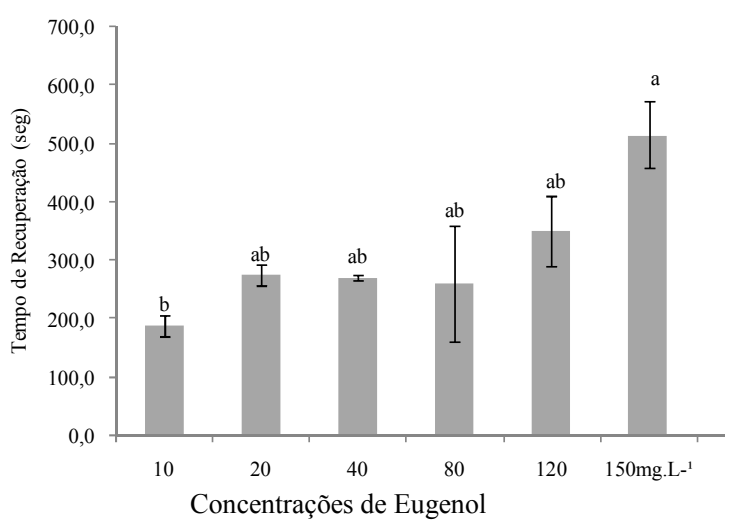

Figura 1. Tempo de indução (a) e recuperação (b) de Carassius auratus submetidos a diferentes concentrações de eugenol

$\mathrm{O}$ aumento na dose de eugenol proporcionou aumento $(\mathrm{P}>0,05)$ no tempo de recuperação a anestesia para $C$. auratus (Figura 1b). A dose de $150 \mathrm{mg}$. $\mathrm{L}^{-1}$ de eugenol proporcionou anestesia rápida dos peixes $(60,0 \pm 10,6$ seg $)$, no entanto, o tempo de recuperação foi de $740 \pm 57,4$ seg. O tempo de recuperação recomendado para o Epinephelus bruneus a indução à anestesia profunda deve ser inferior a 600seg (PARK et al., 2008). A indução à anestesia profunda deve levar de 1 a 3 min e a recuperação não deve ultrapassar 5min (PEREIRADA-SILVA et al., 2009). Ao expor pampo Trachinotus marginatus, à concentração de $25 \mathrm{ppm}$ eugenol os peixes levaram10min para atingir a latência total, e na concentração de $75 \mathrm{ppm}$ apenas $2 \mathrm{~min}$, sendo inversamente proporcional ao tempo de recuperação (OKAMOTO et al., 2009). Em um estudo com $C$. auratus, o tratamento com dose de $75 \mathrm{mg}$.L- ${ }^{1}$ proporcionou tempo de recuperação acima de 15 minutos, inadequado para o bem estar dos peixes (BITTENCOURT et al., 2012).

A indução anestésica de $C$. auratus à diferentes concentrações de eugenol proporcionou elevação $(\mathrm{P}>0,05)$ de $27,5 \%$ nos valores de percentual de hematócrito e de $37 \%$ no número de eritrócitos (Tabela 1). A anestesia com óleo de cravo em tuvira (Gymnotus inaequilabiatus) ocasiona alterações no hemograma, dose-dependente (PADUA et al., 2012). Para o tambaqui (Colossoma macropomum) o uso de óleo de cravo promoveu aumento na quantidade de eritrócito (PADUA et al., 2013). Apesar do aumento na quantidade de eritrócito em $C$. auratus estas caracterizam-se como normocíticasnormocrômicas, resultante da ação de catecolaminas (liberadas pela ativação do eixo hipotálamo - sistema nervoso simpático - células cromafins), que determinam a contração esplênica em resposta ao estímulo de estresse agudo (INOUE et al., 2004). Estes corroboram 
Rev. Bras. Saúde Prod. Anim., Salvador, v.17, n.3, p.545-552 jul./set., $2016 \quad$ http://www.rbspa.ufba.br

com a elevação dos níveis de glicose plasmática em resposta ao banho anestésico com eugenol em relação ao grupo controle (Tabela 1). A glicose é um dos indicadores mais utilizados na avaliação do estresse em peixes como resposta secundária, sua elevação devese a presença de algum fator estressante para suprir a maior demanda energética, característica de situações desfavoráveis
(DINIZ \& HONORATO, 2012). O uso de anestésico pode ter efeitos secundários indesejados que reduzem o bem-estar dos peixes, como a acidose (ZAHL et al., 2012). Neste estudo não observou-se o quadro de acidose metabólica uma vez que os valores de $\mathrm{pH}$ e bicarbonato plasmático sofreram alterações em relação ao grupo controle (Tabela 1).

Tabela 1. Parâmetros sanguíneos de $C$. auratus submetidos a diferentes concentrações de eugenol

\begin{tabular}{|c|c|c|c|c|c|c|}
\hline \multirow{2}{*}{ Parâmetros } & \multirow{2}{*}{ Controle } & \multicolumn{5}{|c|}{ Concentração de eugenol (g.L-1) } \\
\hline & & 20 & 40 & 80 & 120 & 150 \\
\hline \multicolumn{7}{|l|}{ Eritrograma } \\
\hline $\begin{array}{l}\text { Eritrocito } \\
(\mathrm{x} 106 \mu \mathrm{L}-1)\end{array}$ & $2,9 \pm 0,5^{\mathrm{b}}$ & $3,2 \pm 0,5^{\mathrm{ab}}$ & $3,7 \pm 0,4^{\mathrm{b}}$ & $3,6 \pm 0,6^{\mathrm{a}}$ & $3,5 \pm 0,5^{\mathrm{a}}$ & $3,7 \pm 0,4^{\mathrm{a}}$ \\
\hline $\begin{array}{l}\text { Hemoglobina } \\
\text { (g.dL-1) }^{-1}\end{array}$ & $12 \pm 1,5$ & $13,6 \pm 1,6$ & $11,5 \pm 1,4$ & $17,4 \pm 2,1$ & $13,6 \pm 1,6$ & $11 \pm 1,3$ \\
\hline Hematócrito (\%) & $36 \pm 3,7^{\mathrm{b}}$ & $39,3 \pm 4,0^{\mathrm{ab}}$ & $38,8 \pm 3,4^{\mathrm{b}}$ & $49,5 \pm 5,0^{\mathrm{a}}$ & $40,4 \pm 4,1^{\mathrm{a}}$ & $41 \pm 3,2^{\mathrm{a}}$ \\
\hline VCM (f.L) & $123,3 \pm 24,3$ & $123,6 \pm 24,3$ & $126,1 \pm 24,8$ & $138,7 \pm 27,3$ & $115,1 \pm 22,7$ & $116,5 \pm 23,0$ \\
\hline $\mathrm{HCM}(\mathrm{pg})$ & $41,1 \pm 6,0$ & $42,8 \pm 6,2$ & $42,8 \pm 6,2$ & $48,7 \pm 7,1$ & $38,7 \pm 5,7$ & $41,4 \pm 6,0$ \\
\hline CHCM (g.dL-1 $)$ & $33,3 \pm 4,7$ & $34,6 \pm 4,9$ & $33,9 \pm 4,8$ & $35,2 \pm 5,0$ & $33,7 \pm 4,8$ & $35,5 \pm 5,0$ \\
\hline \multicolumn{7}{|c|}{ Controle ácido-base } \\
\hline $\mathrm{pH}$ & $7,3 \pm 0,6^{\mathrm{a}}$ & $7,2 \pm 0,6^{\mathrm{a}}$ & $6,8 \pm 0,6^{\mathrm{ab}}$ & $6,8 \pm 0,6^{\mathrm{ab}}$ & $6,7 \pm 0,6^{b}$ & $6,7 \pm 0,6^{b}$ \\
\hline Bicarbonato & $4,6 \pm 0,2^{\mathrm{c}}$ & $5,9 \pm 0,2^{\mathrm{b}}$ & $5,9 \pm 0,3^{\mathrm{a}}$ & $5,1 \pm 0,2^{\mathrm{a}}$ & $4,9 \pm 0,2^{\mathrm{ab}}$ & $4,6 \pm 0,2^{\mathrm{a}}$ \\
\hline \multicolumn{7}{|c|}{ Metabólito secundário } \\
\hline Glicose (g.dL-1) & $99 \pm 9,7^{b}$ & $263 \pm 19,7^{\mathrm{a}}$ & $246 \pm 19,5^{\mathrm{a}}$ & $242 \pm 18,2^{\mathrm{a}}$ & $254 \pm 17,9^{\mathrm{a}}$ & $257 \pm 18,8^{\mathrm{a}}$ \\
\hline
\end{tabular}

O estudo da variável bicarbonato plasmático $\left(\mathrm{HCO}_{3}{ }^{-}\right)$permite avaliar a resposta do organismo frente a variações do $\mathrm{pH}$ no sangue.O bicarbonato é responsável por mais de $50 \%$ da capacidade tampão extracelular (LUNA, 2002). Neste estudo observouse aumento na concentração de bicarbonato plasmático até a concentração de $40 \mathrm{mg} \cdot \mathrm{L}^{-1}$. Estes estão diretamente relacionados a elevação na $\mathrm{PaCO}_{2}$ pois o mecanismo compensatório eleva as taxas de bicarbonato a fim de neutralizar o excesso de $\mathrm{CO}_{2}$ presente no sangue. Nas concentrações acima de $80 \mathrm{mg} . \mathrm{L}^{-1}$ observou-se manutenção nos níveis de bicarbonato que podem ser atribuídas ao aumento dos batimentos operculares comuns no primeiros estágios anestésicos destas concentrações de $C$. auratus quando submetidos ao aumento de concentração de eugenol como indutor anestésico apresentaram uma sutil diminuição na $\mathrm{PaO}_{2}$, e leve aumento na $\mathrm{PaCO}_{2}$ (Figura 2).

A diminuição do $\mathrm{pH}$ com concomitante aumento de bicarbonato (Tabela 1), revela que o peixe submetido a maiores concentrações de eugenol apresentou 
quadro de acidose respiratória. $\mathrm{O}$ aumento de $\mathrm{CO}_{2}$ desencadeia a reação de formação de ácido carbônico $\left(\mathrm{H}_{2} \mathrm{CO} 3\right)$, que posteriormente pela ação da anidrase carbônica aumenta a produção de bicarbonato (HCO3), que

a)

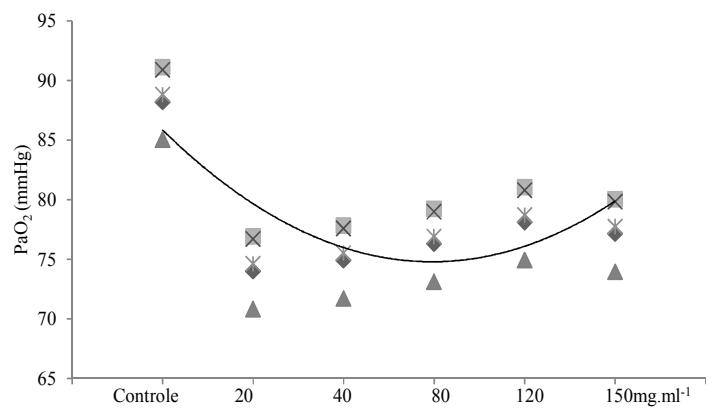

atua como tampão fisiológico. Estes resultados permite estabelecer o quadro de acidose respiratória caracterizada pela diminuição do $\mathrm{pH}$, decréscimo nos teores de $\mathrm{HCO}_{3}{ }^{-}$e aumento da $\mathrm{PaCO}_{2}$.

Figura 2. (a) Pressão parcial de oxigênio $\left(\mathrm{PO}_{2}\right)$ e (b) dióxido de carbono $\left(\mathrm{PCO}_{2}\right)$ de $C$. auratus submetidos a diferentes concentrações de eugenol

A utilização de anestésicos pode causar reações adversas secundárias tais como acidose e estresse osmótico devido à parada respiratória e insuficiente troca gasosa e iônica entre o sangue e a água (ZAHL et al., 2012). As variações $\mathrm{PaO}_{2} \mathrm{e}$ $\mathrm{PaCO}_{2}$ podem ser compensadas por compensações cardíacas (KEEN et al., 2012). Os peixes apresentarem hiperventilação quando estão no estagio III de anestesia, com o intuito de aumentar as trocas gasosas. No entanto, para $\mathrm{o} C$. auratus aumento dos batimentos operculares não foi suficiente para aumentar a atividade cardiorespiratória resultando em baixas trocas gasosas.

A difusão das trocas gasosas nas brânquias dos teleósteos é mais dificultada pela excreção de $\mathrm{CO}_{2}$ do que pela captação de $\mathrm{O}_{2}$ (LIMA BOIJINK et al., 2010). Mecanismo de hiperventilação são respostas ao decréscimo na concentração de oxigênio no sangue (hipoxemia) induzida pela acidose
(KEEN et al., 2012) em contrapartida a elevação na concentração de $\mathrm{CO}_{2}$ no sangue é capaz de desencadear respostas cardiorrespiratórias (PORTEUS et al., 2012). No entanto, o aumento na pressão de $\mathrm{CO}_{2}$ arterial, e concomitante diminuição do $\mathrm{pH}$, são inevitáveis durante a hipercardia, mecanismo este utilizado para alterar o gradiente de difusão entre o sangue e água diminuindo a $\mathrm{PCO}_{2}$ (MILSON, 2012).

A utilização do eugenol possibilita o manejo nas pisciculturas, a dose recomendada é de $40 \mathrm{mg}$. $\mathrm{L}^{-1}$ para Carassius auratus sem comprometimento das funções cardiorrespiratórias

\section{AGRADECIMENTOS}

A Dra. Fernanda P. L. Zauith Diretora do Hospital Veterinário da UNIGRAN.O estudo foi aprovado pela comissão de bioética e foi realizado de acordo com as normas técnicas de biossegurança e ética. Processo CEUA-104/11. 
Rev. Bras. Saúde Prod. Anim., Salvador, v.17, n.3, p.545-552 jul./set., $2016 \quad$ http://www.rbspa.ufba.br

\section{REFERÊNCIAS}

BITTENCOURT, F.; SOUZA, B.E.; BOSCOLO, W.R.; RORATO, R.R.; FEIDEN, A.; NEU, D.H. Benzocaína e eugenol como anestésicos para o quinguio (Carassiusauratus). Arquivo Brasileiro de Medicina Veterinária e Zootecnia, v.64, n.6, p.1597-1602, 2012.

COLLIER, H.B. The standardizations of blood haemoglobin determinations.

Canadian Medical Association Journal, v.50, n.6, p.550-552, 1944.

DIEMER, O.; NEU, D.H.; BITTENCOURT, F.; SIGNOR, A.; BOSCOLO, W.R.; FEIDEN, A. Eugenol como anestésico para jundiá (Rhamdiavoulezi) em diferentes pesos. Semina: Ciências Agrárias, v.33, n.4, p.1495-1500, 2012.

DINIZ, N.M.; HONORATO, C.A. Algumas alternativas para diminuir os efeitos do estresse em peixes de cultivo - revisão. Arquivo de Ciências Veterinária e Zoologia UNIPAR, v.15, n.2, p.149-154, 2012.

GHOLIPOURKANANI, H.; AHADIZADEH, S. Use of propofol as an anesthetic and its efficacy on some hematological values of ornamental fish Carassius auratus. Springer Plus, v.76, n.2, p.1-5, 2013.

GOLDENFARB, P.B.; BOWYER, F.P.; HALL, E.; BROSIOUS, E.

Reproducibility in the hematology laboratory: the microhematocrit determinations. American Journal of Clinical Pathology, v.56, n.1, p.35-39, 1971.

INOUE, L.A.K.A.; HACKBARTH, A.; MORAES, G. Assessment of 2phenoxyethanol and benzocaine as anesthetics for field procedures in matrinxa (Bryconcephalus).

Biodiversidade Pampeana, v.2, n.1, p.10-15, 2004.

INOUE, L.A.K.; BOIJINK, C.L.; RIBEIRO, P.T.; SILVA, A.M.D.; AFFONSO, E.G. Avaliação de respostas metabólicas do tambaqui exposto ao eugenol em banhos anestésicos. Acta Amazonica, v.41, n.2, p.327-332, 2011.

KEEN, A.N.; GAMPERL, A.K. Blood oxygenation and cardio respiratory function in steelhead trout (Oncorhynchus mykiss) challenged with an acute temperature increase and zatebradine-induced bradycardia.

Journal of Thermal Biology, v.37, n.3, p.201-210, 2012.

LIMA BOIJINK, C.; FLORINDO, L.H.; LEITE, C.A.C.; KALININ, A.L.; MILSOM, W.K.; RANTIN, F.T. Hypercarbic cardiorespiratory reflexes in the facultative air-breathing fish jeju (Hoplerythrinusunitaeniatus): the role of branchial $\mathrm{CO}_{2}$ chemoreceptors.

Journalof Experimental Biology, v.213, p.2797-2807, 2010.

LUNA, S.P.L. Equilíbrio acido-básico. In: FANTONI, D.T.; CORTOPASSI, S.R.G. Anestesia em cães e gatos. São Paulo: Rocca, 2002. p.120-129.

MAZZAFERA, P. Efeito alelopático do extrato alcoólico do cravo-da-índia e eugenol. Revista Brasileira de Botânica, v.26, n.2, p.231-238, 2003.

MILSON, W.K. New insights into gill chemoreception: receptor distribution and roles in water and air breathing fish.

\section{Respiratory Physiology \&}

Neurobiology, v.184, n.3, p.326-339, 2012. 
Rev. Bras. Saúde Prod. Anim., Salvador, v.17, n.3, p.545-552 jul./set., $2016 \quad \underline{\text { http://www.rbspa.ufba.br }}$ ISSN 15199940

OKAMOTO, M.H.; TESSER, M.B.; LOUZADA, L.R.; SANTOS, R.A.; SAMPAIO, L.A. Benzocaína e eugenol como anestésicos para juvenis do pampo Trachinotusmarginatus. Ciência Rural, v.39, n.3, p.866-877, 2009.

PÁDUA, S.B.; DIAS NETO, J.;

SAKABE, R.; CLAUDIANO, G. da S.; CHAGAS, E.C.; PILARSKI, F. Notas Científicas Variáveis hematológicas em tambaquis anestesiados com óleo de cravo e benzocaína. Pesquisa

Agropecuária Brasileira, v.48, n.8, p.1171-1174, 2013.

PADUA, S.B.; VENTURA, A.S.; SATAKE, F.; ISHIKAWA, M.M.; HISANO, H.; ROTTA, M.A.; ARANTES, F.C. Respostas hematológicas em tuvira após anestesia com diferentes concentrações de óleo de cravo. Boletim do Instituto de Pesca, v.38, n.3, p.181-188, 2012.

PARK, M.O.; HUR, W.J.; IM, S.T.; SEOL, D.W.; LEE, J.; PARK, I.S.

Anaesthetic efficacy and physiological responses to clove oil anaesthetized kelp grouper Epinephelusbruneus.

Aquaculture Research, v.39, p.877-884, 2008.

PEREIRA-DA-SILVA, E.M.; OLIVEIRA, R.H.F.; RIBEIRO, M.A.R.; COPPOLA, M.P. Efeito anestésico do óleo de cravo em alevinos de lambari. Ciência Rural, v.39, n.6, p.1851-1856, 2009.

PORTEUS, C.S.; BRINK, D.L.; MILSOM, W.K. Neurotransmitter profiles in fishgills: Putative gill oxygen receptors. Respiratory Physiology \& Neurobiology, v.184, n.3, p.316-325, 2012.

ROSS, L.G.; ROSS, B. Anaesthetic and sedative techniques for aquatic animals. 3.ed. Oxford: Blackwell, 2008. $222 p$.
ROTILI, D.A.; DEVENS, M.A.;

DIEMER, O.; LORENZ, E.K.;

LAZZARI, R.; BOSCOLO, W.R. Uso

de eugenol como anestésico em pacu.

Pesquisa Agropecuária Tropical, v.42, n.3, p.288-294, 2012.

TAGHIZADEH, V.; IMANPOOR, M.; HOSSEINZADEH, M.; AZARIN, H. Effects of acidic water in combination with aluminum on swimming behavior and survival of yolk-sac larval in Goldfish (Carassius auratus gibelio ). SpringerPlus, v.2, n.1, p.1-6, 2013.

TRINDER, P. Determination of glucose in blood using glucose oxidase with an alternative oxygen acceptor. Analytical Clinical Biochemistry, v.6, p.24-27, 1969.

VIDAL, L.V.O.; ALBINATI, R.C.B.; ALBINATI, A.C.L.; LIRA, A.D.; ALMEIDA, T.R.; SANTOS, G.B. Eugenol como anestésico para a tilápiado-Nilo. Pesquisa Agropecuária Brasileira, v.43, n.8, p.1069- 1074, 2008.

WINTROBE, M.M. Variations in the size and hemoglobin content of erythrocytes in the blood of various vertebrates. Folia Haematologica, v.51, p.32-49, 1934.

ZAHL, I.; SAMUELSEN, O.; KIESSLING, A. Anaesthesia of farmed fish: implications for welfare. Fish Physiology and Biochemistry, v.38, n.1, p.201-218, 2012.

Data de recebimento: 25/03/2015

Data de aprovação: 20/07/2016 